\title{
Die fünf Daseinsgruppen (skandhas) in Abhebung zu Platons Auffassung der Sinnes- und Seelentätigkeit
}

\author{
Thomas Auinger
}

This article compares the Buddhist conception of the five skandhas (essential aggregates, which combine to embody an individual) with Plato's philosophical position concerning the epistemic relevance of perception, including the argumentation, which reveals the overall important ontological and categorical realm, corresponding to the instance called psyche. The Buddhistical way leads to the decisive doctrine of non-self (anatman), the platonic way, however, to a theory of soul and self, which determines the whole western tradition. What is true, what is false? On the one hand we may only state a koan (a paradox to be meditated upon); on the other hand we may seek a philosophical position drawing the consequences of both antagonists. The claim is that Hegel's speculative logic is exactly this requested position.

Thomas Auinger is a philosopher, who lectures at the University of Vienna, the FH Hagenberg and the Danube University Krems. Research topics: Connections of (post)analytical and continental philosophy, Hegel's speculative logic, Buddhism and philosophy inspired by Buddhism. He has been a Buddhist religious education teacher since 2015.

\section{Einleitung}

Die Lehre von den sogenannten fünf Haufen oder Anhäufungen, wie der SanskritTerminus skandha ziemlich wörtlich wiedergegeben werden kann, stellt einen zentralen buddhistischen Lehrinhalt dar, welcher sich grundlegend von jenen philosophisch-anthropologischen Konzeptionen unterscheidet, die im abendländischen Kulturkreis ausgebildet wurden.

Es sollen in den folgenden Ausführungen weniger die einzelnen Daseinsgruppen $^{1}$ in ihrer spezifischen Charakteristik untersucht werden, als vielmehr jene zentralen Aspekte deutlich werden, wodurch solch eine Auffassung vom personalistischen Ansatz der europäischen Philosophie (vertreten durch Platon) unterschieden ist. Freilich ließen sich auch in der sogenannten westlichen Hemisphäre Philosophen (und bei genauerer Recherche vielleicht auch Philosophinnen) auffinden, bei welchen bezüglich gewisser Theorieteile manch Ähn-

1 Bei der Erläuterung des Pali-Ausdrucks khandha, der dem Sanskritwort skandha entspricht, wird von Nyanatiloka von „Gruppen“ bzw. „Daseinsgruppen“ gesprochen. Vgl. Nyanatiloka 1999, S. 106-111. 
lichkeiten und Parallelen zur Skandha-Lehre nachgewiesen werden könnten. In erster Linie denke ich dabei an David Hume, auf den ich jedoch hier nicht eingehen werde. Abgesehen von derartigen Detailbezügen gab es jedoch keine Position innerhalb der abendländischen Philosophiegeschichte, die auch nur annähernd eine Konzeption entwickelt hätte, die mit der buddhistischen SkandhaLehre wesentliche Gemeinsamkeiten aufweisen würde. Ganz im Gegenteil gibt es eine Vielzahl philosophischer Richtungen, die in äußerst dezidierter Form vom buddhistisch anti-substantialistischen Menschenbild, und auch Weltbild, ${ }^{2}$ abweichen. Das ist insofern nicht verwunderlich, weil ja das Konzept der Substanz (griechisch ousía) von Aristoteles, jenem auf allen Gebieten einflussreichsten antiken Gelehrten, entwickelt und in einer ganzen Reihe von maßgeblichen Texten (insbesondere in seiner prōtē philosophia, der später sogenannten Metaphysik) ausgearbeitet wurde. Bei diesem ganz allgemein gehaltenen Hinweis möchte ich jedoch für den vorliegenden Kontext nicht stehenbleiben, sondern genauere Gründe dafür angeben, warum im westlichen Philosophieverständnis nie eine vergleichbare Auffassung hat entstehen können. Zu diesem Zweck werde ich mich zunächst Platon zuwenden, der eine bestimmte Argumentation eingeführt hat, die für all seine philosophischen Nachfolger eminent wichtig geworden ist, selbst dann, wenn sie versucht haben, sich möglichst radikal vom platonischen Denkhorizont zu entfernen.

Bevor ich jedoch mit meinen Ausführungen zu Platon beginne, seien zumindest in Kurzform die fünf Daseinsgruppen angeführt und konzis erläutert, damit ersichtlich wird, wie sich bestimmte Anschauungen der griechischen Philosophie davon unterscheiden.

\section{Skandhas - Bestimmung der „fünf Haufen“}

Die Erscheinungsweise menschlicher Individualität ist ein Bedingungsgefüge, das durch das Zusammenkommen von fünf funktionalen Anhäufungen zustande kommt. In keinem dieser Bereiche, auch nicht in deren Unterbestimmungen, findet sich etwas Subsistierendes, das ein essentielles Fürsichsein begründen könnte.

Diese fünf Gruppen haben überdies ein hohes Anhaftungspotential, weil sie Identifikationen ermöglichen, die dazu führen, fälschlicherweise selbständige Entitäten anzunehmen. Es findet sich jedoch nirgends eine substantiierte persönliche Egoität oder Ichheit.

Nyanatiloka schreibt hierzu:

2 „Denn die kosmische Totalität und die psychomentale Aktivität bilden ein und dasselbe Universum. “ Eliade 1979, S. 88 f. 
Unser sogenanntes individuelles Dasein ist, genau so wie alles Dasein überhaupt, in Wirklichkeit nichts weiter als ein bloßer Prozeß dieser körperlichen und geistigen Phänomene, ein Prozeß, der seit undenkbarer Zeit schon vor unserer Geburt im Gange war und der auch noch nach dem Tode sich für undenkbar lange Zeitperioden fortsetzen wird. Diese fünf Gruppen aber bilden weder einzeln noch zusammengenommen irgendeine in sich abgeschlossene wirkliche Ich-Einheit, und auch außerhalb derselben existiert nichts, was man als eine für sich unabhängig bestehende Ichheit bezeichnen könnte, so daß eben der Glaube an eine im höchsten Sinne wirkliche Ichheit, Persönlichkeit usw. eine bloße Illusion ist. $^{3}$

Die fünf Dharma-Anhäufungen lauten wie folgt:

\section{Körper (rūpa)}

Hierzu zählen alle Bestandteile, Eigenschaften und Dispositionen der Körperlichkeit. In Bezug auf die Wahrnehmungsfähigkeit sind insbesondere die physischen Sinnesorgane zu erwähnen. Alle weiteren Glieder des Organismus kommen ebenso in Betracht. In den Suttas werden einunddreißig Körperteile angeführt. ${ }^{4}$

Dieses erste Skandha wird oft auch mit dem Terminus Form angesprochen. So etwa im Herzsutra (= Mahaprajnaparamita-Hridaya-Sutra, japanisch Hannya Shingyō), wo die für das $\mathrm{Zen}^{5}$ so wichtige Stelle vorkommt:

Form ist nichts anderes als Leere und Leere nichts anderes als Form $;^{6}$

Danach wird in diesem Sutra gesagt, dass es in der Leere auch die anderen Skandhas nicht gebe. Hierin bezeugt sich der tiefgründig weisheitsvolle ZenGeist, in welchem alle lehrhaften Klassifikationen transzendiert werden. Wir dürfen dies jedoch keineswegs als Leugnung derartiger Lehrinhalte verstehen. Vielmehr handelt es sich dabei um ein nicht-diskursives, die Beschränkungen formaler Logik übersteigendes Verstehen.

Als Grundbausteine alles Körperlichen gelten die vier Elemente bzw. die mit ihnen verbundene jeweilige Charakteristik. ${ }^{7}$ Insgesamt unterscheidet die buddhistische Tradition vierundzwanzig körperliche Phänomene.

3 Nyanatiloka 1981, S. 176.

4 So etwa im Satipatthana-Sutta (Majjhima Nikaya 10).

5 Vgl. Izutsu 1979.

6 Vgl. Aitken 1998, S. 155 f.

7 „Weiter sodann, ihr Mönche: der Mönch schaut sich diesen Körper da wie er geht und steht als Artung an: ,Dieser Körper ist von Erdenart, von Wasserart, von Feuerart, von Luftart.““ Ebd., Satipatthana-Sutta (Majjhima Nikaya 10). 


\section{Gefühl (vedanā)}

Die Berührung mit den Objekten, im Sinne einer Reaktion auf gewisse Reize, löst bestimmte Gefühle, Empfindungen aus. Spezifisch buddhistisch ist die Einteilung der Gefühlsregungen in angenehme, unangenehme und neutrale.

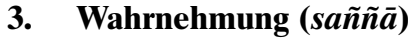

Hier ist wichtig zu beachten, dass dezidiert sechs Sinne unterschieden werden: Sehen, Hören, Riechen, Schmecken, Tasten und Denken. Geistige Aktivitäten zählen hiermit auch zur Sphäre der Sinnlichkeit. Am besten spricht man in diesem Zusammenhang von einem Gewahrwerden, worin sich beispielsweise Farben, Töne, Gerüche, Geschmackseindrücke, Bilder, Vorstellungen und Gedankeninhalte manifestieren. Die Wahrnehmungssphäre ist zwar komplexer als die Gefühlssphäre, es bleibt jedoch bei der Parallelität von Sehen und Seheindruck, Hören und Höreindruck usw. Das ist ein Punkt, der bei der Besprechung der platonischen Bestimmung des Wahrnehmens von zentraler Bedeutung sein wird.

Im Bereich der Wahrnehmungen wird auch das Phänomen der Täuschung explizit. Es eröffnet sich die Frage, worin eine fehlerhafte Wahrnehmung überhaupt besteht. Auch diese Fragestellung hat einen Bezug zur platonischen Sichtweise.

\section{Geistesformationen (sankhära)}

Es gibt hier zahlreiche Ausdrücke, um diesen Bereich klarer zu charakterisieren. Neben dem Terminus Geistesformation ist auch von Gestaltung, Strebung, Impuls, Interesse, Willensregung, Sehnsucht, Absicht usf. die Rede. Es sind dies alles Tendenzen, die das Potential haben, zu bestimmten Handlungen zu führen.

Bei diesem dritten Skandha wird sehr deutlich, wie schnell sich hier Identifikationsmuster und Anhaftungsphänomene einstellen können.

\section{Bewusstsein (viññāna)}

Hierbei ist äußerst wichtig, dass der sechsfachen Sinnestätigkeit ganz parallel das Seh-, Hör-, Riech-, Schmeck-, Tast- und Denkbewusstsein zugeordnet wird.

Wir werden sofort sehen, wie sich Platons Auffassung entschieden hiervon abhebt. 


\section{Platons ontologisch orientierte Auffassung der Sinnes- und Seelentätigkeit}

Schon bei den Vorgängern Platons, den vorsokratischen Naturphilosophen, lässt sich eine Vereinheitlichungstendenz beobachten, indem sie nämlich nach dem Urprinzip (griechisch arché) fragen, das allem Seienden und dem Sein selbst zugrunde liegen soll. Zunächst wurde diese Frage durch die Angabe von stofflichen Prinzipien beantwortet, später wurden die diesbezüglichen Überlegungen subtiler, abstrakter, aber auch, falls es einen diesbezüglichen Komparativ geben sollte, philosophischer. Sehr interessant sind die (leider nur fragmentarisch erhaltenen) Ausführungen von Anaximander und Anaxagoras. Ersterer spricht vom Unbegrenzten (griechisch apeiron), letzterer vom Denken bzw. (in für diese Frühzeit besserer Wiedergabe) vom Vernehmen oder Begreifen (griechisch nous), das als Seinsprinzip fungieren sollte. Abgesehen von der sehr diffizilen Interpretation dieser Bestimmungen, worum sich insbesondere Heidegger große Verdienste erworben hat, wird doch ersichtlich, dass schon in dieser frühen Periode der griechischen Philosophie ein ganz großes Augenmerk auf das prinzipielle Seinsverstehen oder Seinsverständnis gelegt worden ist.

Diese Perspektive und Fragerichtung ändert sich auch bei Platon nicht, er führt jedoch in Bezug auf die philosophisch verhandelten Themen bei weitem ausführlichere und durch die zumeist gewählte Dialogform äußerst differenzierte Argumentationen an. Die Auslegung dieser argumentativen Strukturen bleibt nach wie vor eine große hermeneutische Aufgabe, woraus sich jedoch auch in Zukunft immer noch neuartige philosophische Einsichten ergeben werden.

Um den gravierenden Unterschied zur Skandha-Lehre klar aufzeigen zu können und um dadurch gleichzeitig ein besseres Verständnis für dieses so wichtige buddhistische Lehrstück zu gewinnen, möchte ich genauer auf einen bestimmten Abschnitt im platonischen Dialog Theaitetos eingehen. Hierfür wird es erforderlich sein, etwas längere Passagen zu zitieren. Gerade bei Platons so eigenwilliger Darstellungsweise im Stil der Rede und Gegenrede, sind zu kurz gehaltene Zitate häufig entweder missverständlich oder überhaupt unverständlich. Das soll hier nicht passieren.

Das Generalthema des Dialogs Theaitetos ist die philosophische Kardinalfrage: Was ist Erkenntnis (griechisch epistéme)? In manchen Übersetzungen wird der griechische Terminus auch mit Wissen wiedergegeben, für unsere Zwecke besitzt das keine Relevanz.

Im Hauptteil behandelt der Dialog drei Thesen oder Behauptungen, die einer eingehenden Untersuchung zugeführt werden. Platon beschließt den Dialog mit keiner eindeutigen Festlegung, dennoch hat die analytische Philosophie ihre Wissensdefinition (knowledge is justified true belief, JTB) von der letzten verhandelten Position in diesem Dialog abgeleitet.

Für uns ist ein bestimmter Abschnitt in der von Sokrates angeleiteten Analyse der ersten These von zentraler Bedeutung. Diese These, im Dialog vom jungen 
Theaitetos vorgebracht, besagt, dass Erkenntnis mit Wahrnehmung (griechisch aísthēsis) gleichgesetzt werden könne:

Sokrates: Versuche also noch einmal von Anfang an, o Theaitetos, zu sagen, was Erkenntnis ist. Daß du aber nicht kannst, sage nur niemals. Denn so Gott will und du wacker bist, wirst du es wohl können.

Theaitetos: Wenn du freilich, Sokrates, solchergestalt zuredest, wäre es schändlich nicht auf alle Weise mutig zu sagen, was einer eben hat. Mir also scheint, wer etwas erkennt, dasjenige wahrzunehmen, was er erkennt; und wie es mir jetzt erscheint, ist Erkenntnis nichts anderes als Wahrnehmung.

Sokrates: Gut und wacker, Jüngling. So muß sich deutlich machen, wer etwas erklärt. Wohlan laß uns nun dieses gemeinschaftlich betrachten, ob es eine rechte Geburt ist oder ein Windei. Wahrnehmung, sagst du, sei Erkenntnis.

Theaitetos: Ja. ${ }^{8}$

In die hierauf folgende Auslegung und kritische Untersuchung dieser Behauptung ist in erster Linie die Diskussion des protagoräischen „Homo-MensuraSatzes“9 eingeflochten. Wenn der Mensch das Maß aller Dinge ist, hat dies zur Konsequenz, dass alle Eindrücke, die sich für jemanden wahrnehmungsmäßig einstellen, einen erkenntnisdignitären oder wissensmäßigen Stellenwert haben. Ich weiß etwa darum, dass ein bestimmter Windstrom in meiner spezifisch menschlichen Auffassungs- bzw. Wahrnehmungsweise als kühl erscheint. Im Sinne einer derartig unmittelbar verstandenen Wissensbestimmung kann niemand an meinem sich in eben dieser Weise einstellenden Eindruck zweifeln. Es ist einfach für mich wahr und darüber lässt sich nicht streiten. Damit wird aber die Wahrheit versubjektiviert, denn für mich ist der Kühlheitseindruck wahr, für jemand anderen wirkt jedoch ein und derselbe Windstrom warm. Das mag zunächst unproblematisch sein, und diese Sichtweise wird, insbesondere von philosophischen Laien und Anfängern, sehr gerne vertreten. Man solle in der Wahrheitsbestimmung nicht so streng und elitär sein, eher jedem seine eigene Wahrheit lassen. Für mich ist dies wahr, für jemand anderen vielleicht das Gegenteil, das kann, soll und darf doch nebeneinander bestehen.

Genau diese Art der Wahrheitsbeliebigkeit und die durch die Wahrnehmungsthese auf die spezifisch menschliche Subjektivität zugeschnittene Erkenntnisauffassung wird von Platon kritisiert und im typisch sokratischen Stil so widerlegt, dass der Gesprächspartner selbst von der Unrichtigkeit der Anfangs-

\footnotetext{
8 Dialog ,Theaitetos“ in Platon 1990, 151d-151e (= S. 31).

9 Zum „Homo-Mensura-Satz“ des Protagoras heißt es bei Diogenes Laertius: „Eine seiner Schriften fing mit folgenden Worten an: ,Der Mensch ist das Maß aller Dinge, der seienden, daß sie sind, der nicht seienden, daß sie nicht sind. ' Er behauptete, die Seele bestehe lediglich aus den sinnlichen Wahrnehmungen [...] und alles sei wahr." Diogenes Laertius 1998, S. 186.
} 
bestimmung - hier die Gleichsetzung von Wahrnehmung und sich darin einstellender untrüglicher Erkenntnis ${ }^{10}$ - überzeugt ist.

Es ist für die Zwecke der Abhebung von der Skandha-Lehre nicht erforderlich, auf alle einzelnen Argumentationsschritte einzugehen. Platon diskutiert via Sokrates ausführlich Pro- und Contra-Argumente, bis er dann zur endgültigen Widerlegung ansetzt. Zentral ist hierbei das Abzielen auf einen Vereinheitlichungspunkt und die erwähnte ontologische Darlegungsstrategie.

Sokrates: So erwäge denn, o Theaitetos, was das bisher Gesagte betrifft, auch noch dieses. Wahrnehmung sei Erkenntnis, hattest du geantwortet. Nicht wahr?

Theaitetos: Ja.

Sokrates: Wenn nun jemand dich so fragte: Womit doch sieht der Mensch das Weiße und Schwarze, und womit hört er das Hohe und Tiefe, würdest du, glaube ich, sagen: Mit den Augen und Ohren.

Theaitetos: Ich gewiß.

Sokrates: Es mit Worten aller Art nicht so genau nehmen, und sie nicht mit Spitzfindigkeit aussondern, das ist größtenteils gar nicht unfein, sondern vielmehr das Gegenteil davon hat etwas Unfreies und Knechtisches, nur ist es bisweilen doch notwendig. So ist es auch jetzt nötig, die Antwort, die du gegeben hast, dabei anzugreifen, inwiefern sie nicht richtig ist. Denn betrachte selbst, welche Antwort richtiger ist, ob das, womit wir sehen, die Augen sind, oder das vermittelst dessen, und das, womit wir hören, die Ohren, oder das vermittelst dessen?

Theaitetos: Vermittelst dessen wir jegliches wahrnehmen, dünkt mich besser, als womit. Sokrates: Arg wäre es auch, Sohn, wenn diese mancherlei Wahrnehmungen wie im hölzernen Pferde in uns nebeneinanderlägen, und nicht alle in irgendeinem, du magst es nun Seele oder wie sonst immer nennen, zusammenliefen, mit der wir dann vermittelst jener, daß ich so sage, Werkzeuge wahrnehmen, was nur wahrnehmbar ist.

Theaitetos: Darum dünkt mich auch dieses besser als jenes. ${ }^{11}$

Eine ganz einfache Überlegung führt an dieser Stelle zu einer folgenschweren Festlegung bzw. Einsicht, sofern wir eben Platons weitere Schlüsse daraus für plausibel und zustimmungswürdig halten. Das bleibt, auch angesichts der dagegen stehenden Skandha-Auffassung, immer offen und hinterfragungswürdig.

10 „Wahrnehmung ist also wohl immer des Seienden und untrüglich, wenn sie ja Erkenntnis ist.“ Platon 1990, 152c (= S. 33). Äußerst wichtig ist die eigenwillige Formulierung „des Seienden“, worin sich nämlich der von mir betonte ontologisch-orientierte Argumentationscharakter bekundet. Dadurch wird schon anhand der Kennzeichnung der protagoräischen Position die Ausrichtung an der kategorial verstandenen Seinsdimension in den Vordergrund gestellt. Die allererste Unterscheidungshinsicht ist immer jene von Sein und Nichtsein. Darüber hinaus kommen auch stets weitere kategorielle Hinsichten in Betracht, Platon erwähnt häufig Ähnlichkeit-Unähnlichkeit, Einerleiheit-Verschiedenheit, Größenbestimmungen, Zahlbestimmungen etc. Im hier verwendeten Wortgebrauch zählt all dies zur Ontologiesphäre.

11 Ebd., 184b-184d (= S. 131). 
Weil Sokrates darauf aufmerksam macht, dass es ja eigentlich nicht die Sinnesorgane selbst sind, wodurch sich sinnliche Eindrücke ergeben, sondern sie eher nur als Wahrnehmungswerkzeuge im Sinne von Ermöglichungsinstanzen zu begreifen sind, die ja letztlich nur dazu führen, einen sinnlichen Eindruck erhalten zu können, fokussiert sich das Resultat aller sinnlichen Rezeption auf einen Einheitsort, den Platon als Seele, oder welchen Terminus man hierfür auch immer verwenden will, bezeichnet.

Wichtig ist, dass der Ausdruck Seele als jene Instanz zu begreifen ist, die es in ihrer Einheitlichkeit vermag, das Gemeinschaftliche aller sinnlich erfahrbaren Dispositionen eines Gegenstandes bzw. Dinges zu bestimmen, weil sie die unterschiedlichen Sinnesqualitäten (Farbe, Ton, Geschmack usw.) einerseits auseinanderhalten und andererseits zugleich auf das Einssein eines Dinges (d. i. die Einheitlichkeit auf der Gegenstandsseite) beziehen kann.

Platons Erläuterung, und das ist ganz entscheidend, verläuft über das Hinweisen auf die ontologisch-kategorialen Bestimmungen, die nötig sind, um die Sinnesbereiche als solche überhaupt in ihrer jeweiligen Charakteristik identifizieren zu können. Er beginnt seine diesbezügliche Argumentationslinie mit der grundsätzlichsten, aber für wohl jeden Gesprächspartner äußerst ungewöhnlichen Frage nach dem Seinsstatus der voneinander (durch ihre spezifisch beschränkte Sinnesbereichseröffnung) abgegrenzten Sinnesqualitäten. Danach lässt er via Sokrates auch noch nach weiteren kategoriellen Dimensionen fragen:

Sokrates: Von dem Tone nun und von der Farbe, denkst du nicht von diesen beiden zuerst dieses, daß sie beide sind?

Theaitetos: Das denke ich.

Sokrates: Nicht auch, daß jedes von beiden vom andern verschieden, mit sich selbst aber einerlei ist?

Theaitetos: Freilich.

Sokrates: Und daß sie beide zusammen zwei sind, jedes von beiden aber eins. Theaitetos: Auch dieses. ${ }^{12}$

Im Zugänglichwerden der sinnlichen Qualitäten von allem Erfahrbarem werden deren kategorielle Dimensionen miterschlossen und diese selbst sind nicht einem besonderen Sinnesorgan und keinem sonstigen besonderen Werkzeug zuordenbar. Die kategorielle Einheits- und Unterschiedsdimension, wodurch die Dinge in ihrer Ganzheit bestimmbar werden, verweist auf jene Selbstbezüglichkeitsinstanz, die sogenannte Seele, in welcher alle Vermögen, sinnlich-körperliche und seelisch-geistige (in impliziter und expliziter Kategorialität) zusammenlaufen.

Konzeptionen, die von nur nebeneinander liegenden Impressionen ausgehen, so wie im hölzernen Pferde, um Platons Ausdruck zu verwenden, sind, sofern wir den sokratischen Argumentationsweg mitgehen können, obsolet:

12 Ebd., 185a-185b (=S. 133). 
Sokrates: Ganz recht. Vermittelst wessen wirkt denn nun dasjenige Vermögen, welches dir das in allen und auch in diesen Dingen Gemeinschaftliche offenbart, womit du von ihnen das Sein oder Nichtsein aussagst, und das, wonach ich jetzt eben fragte? Für dies alles, was für Werkzeuge willst du annehmen, vermittelst deren unser Wahrnehmendes jedes davon wahrnimmt?

Theaitetos: Du meinst ihr Sein und Nichtsein, ihre Ähnlichkeit und Unähnlichkeit, Einerleiheit und Verschiedenheit, ferner ob sie eins sind oder eine andere Zahl. Offenbar begreiffst [sic!] du darunter auch die Frage nach dem Geraden und Ungraden, und was damit zusammenhängt, vermittelst welcher Teile des Körpers nämlich wir dies mit der Seele wahrnehmen.

Sokrates: Ganz vortrefflich, o Theaitetos, folgst du mir; denn dies ist es eben, wonach ich frage.

Theaitetos: Aber, beim Zeus, Sokrates, dies wüßte ich nicht zu sagen, außer daß es mir scheint, als gäbe es überall gar nicht ein solches besonderes Werkzeug für dieses wie für jenes, sondern die Seele scheint mir vermittelst ihrer selbst das Gemeinschaftliche in allen Dingen zu erforschen.

Sokrates: Schön bist du, Theaitetos, und gar nicht, wie Theodoros sagt, häßlich; denn wer so schön spricht, der ist schön und gut. Außerdem aber, daß dieses schön gesagt war, hast du auch mir eine große Wohltat erwiesen, indem du mir über vieles Reden hinweggeholfen hast, wenn es dir einleuchtet, daß einiges die Seele selbst vermittelst ihrer selbst erforscht, anderes aber vermittelst der verschiedenen Vermögen des Körpers. Denn eben dieses war es, was ich selbst meinte, und wovon ich wünschte, du möchtest es auch meinen. Theaitetos: Gar sehr leuchtet es mir ein. ${ }^{13}$

Den endgültigen, von mir als ontologisch bezeichneten Widerlegungsschritt beginnt Platon wieder mit der entscheidenden Frage nach dem Sein. Dabei lässt er sofort Theaitetos konstatieren, dass die Beantwortung dieser Fragestellung allein durch die sich auf sich beziehende Seelentätigkeit möglich ist. Es ist das Vermögen des logos, der Ort des Ortes (in der Terminologie von Kitaro Nishida), die hegelisch konnotierte Begriffsdimension des Begriffs, oder auch die, à la Kant ausgedrückt, Sphäre der transzendentalen Einheit der Apperzeption. Wie immer wir es benennen, ausschließlich hierin eröffnet sich der ontologisch-kategoriale Bestimmungshorizont, der letztlich nötig ist, um, zumindest im Traditionsstrang der abendländischen Philosophie, zu einer adäquaten Interpretation des Wissensbegriffs zu kommen. Vorläufig bleibt es beim negativen Resultat, die Unrichtigkeit der Wahrnehmungsthese klar aufzuzeigen.

Sokrates: Zu welchem von beiden rechnest du nun das Sein? Denn dies ist es doch, was am meisten bei allem vorkommt?

Theaitetos: Zu dem, was die Seele selbst durch sich selbst aufsucht.

Sokrates: Wohl auch so die Ähnlichkeit und Unähnlichkeit, das Einerleisein und das Verschiedensein?

Theaitetos: Ja.

13 Ebd., 185c-186a (= S. 135). 
Sokrates: Und wie das Schöne und Schlechte, das Gute und Böse?

Theaitetos: Auch hiervon besonders dünkt mich die Seele das Verhalten gegeneinander zu erforschen, indem sie bei sich selbst das Geschehene und Gegenwärtige in Verhältnis setzt mit dem Künftigen.

Sokrates: Wohlan denn! Wird sie nicht die Härte des Harten und die Weichheit des Weichen vermittelst des Getastes wahrnehmen?

Theaitetos: Ja.

Sokrates: Aber das Sein von beiden, und was sie sind, und ihre Gegensetzung gegeneinander und das Wirklichsein dieser Entgegensetzung, dies versucht also unsere Seele selbst durch Betrachtung und Vergleichung zu beurteilen.

Theaitetos: In alle Wege.

Sokrates: Nicht wahr, jenes wahrzunehmen, was irgend für Eindrücke durch den Körper zur Seele gelangen, das eignet schon Menschen und Tieren von Natur, sobald sie geboren sind. Allein zu den Schlüssen hieraus auf das Sein und den Nutzen gelangen nur schwer mit der Zeit und durch viele Mühe und Unterricht die, welche überall dazu gelangen? Theaitetos: So ist es allerdings. ${ }^{14}$

Sich die Seinsdimension und die Sphäre der Kategorien ${ }^{15}$ erschließen zu können, bedeutet, die aufgrund eines (einer gewissen Mühe bedürfenden) Erfahrungsund Lernprozesses im Menschen prinzipiell angelegte Wissensmöglichkeit auch tatsächlich zu aktivieren, d.h. über die bloß passive Rezeptivität, Eindrücke gleichsam nur zu registrieren, hinauszugelangen.

Die rein sinnlich disponierte Wahrnehmungsdimension reicht jedoch nicht aus, um das Seinsmäßige und Daseinsmäßige adäquat (seinem wahren Wesen nach) erfassen zu können. Somit ist es aus platonischer Sicht völlig offensichtlich, dass es nicht richtig sein kann, Wahrnehmung und Erkenntnis gleichzusetzen.

Im Dialog geleitet Sokrates den Theaitetos, in mäeutischer Manier, hin zu eben dieser Einsicht:

Sokrates: Kann nun wohl dasjenige das wahre Wesen von etwas erreichen, was nicht einmal sein Dasein erreicht?

Theaitetos: Unmöglich.

Sokrates: Wovon man aber das wahre Wesen nicht erreicht, kann man davon Erkenntnis haben?

Theaitetos: Wie könnte man doch, Sokrates.

Sokrates: In jenen Eindrücken also ist keine Erkenntnis, wohl aber in den Schlüssen daraus. Denn das Sein und das wahre Wesen zu erreichen, ist, wie es scheint, nur durch

14 Ebd., 186a-186c (= S. 137).

15 Wie aus dem oben angeführten Zitat (186b) hervorgeht, zählt Platon hierzu ganz explizit auch den Gegensatz. Der sich aus der Bestimmung des Gegensatzes herauskristallisierende Widerspruch wird später zu einer Zentralkategorie in Hegels spekulativer Logik. Dass diese mit der, so können wir formulieren, ontologischsten Bestimmung, nämlich mit dem reinen Sein, beginnt, lässt sich aufgrund meiner Ausführungen in gewisser Hinsicht als durchaus platonisch bezeichnen. 
diese möglich, durch jene aber unmöglich.

Theaitetos: Das leuchtet ein.

Sokrates: Willst du nun jenes und dieses dasselbe nennen, da beides so große Verschiedenheiten zeigt?

Theaitetos: Das scheint wohl nicht billig.

Sokrates: Welchen Namen nun legst du jenem bei, dem Sehen, Hören, Riechen, Frieren, Warmsein?

Theaitetos: Wahrnehmen nenne ich es. Denn wie anders?

Sokrates: Insgesamt also nennst du dies Wahrnehmung.

Theaitetos: Natürlich.

Sokrates: Welcher, wie wir gesagt haben, nicht verliehen ist bis zum wahren Wesen zu gelangen, da sie ja auch nicht bis zum Sein gelangt?

Theaitetos: Nicht verliehen.

Sokrates: Also auch nicht zur Erkenntnis?

Theaitetos: Nicht füglich.

Sokrates: Auf keine Weise also, o Theaitetos, wäre Wahrnehmung und Erkenntnis dasselbe.

Theaitetos: Es scheint nicht; vielmehr ist es jetzt vollkommen deutlich geworden, daß die Erkenntnis etwas anderes ist als die Wahrnehmung. ${ }^{16}$

Was sich nicht bis zur Seinsbestimmung hin erstreckt, vermag nicht, das wahre Wesen zu berühren oder zu fassen. Dies ist, nunmehr explizit ausgesprochen, Platons ontologische Ansicht in Bezug auf alle genuin philosophischen Fragestellungen.

Im Dialog wird an dieser Stelle die Frage nach der Erkenntnis, was sie sei und wie sie exakt zu bestimmen wäre, erneut aufgerollt. Das ist für uns nicht weiter relevant.

Als Ergebnis gilt es festzuhalten, dass Platon im Zuge der Diskussion des Erkenntnisbegriffs eine Analyse der Sinnlichkeitsdimension gibt, die unvereinbar ist mit der buddhistischen Skandha-Lehre. Es könne kein Nebeneinander der über die Sinne vermittelten Impressionen geben und die mit dem Körper assoziierten rezeptiven Fähigkeiten verweisen notwendig auf einen sie alle vereinenden Einheitspunkt, die sogenannte Seele als Topos der Personalität und IchEinheit, kantisch gesprochen auch der Sitz der Spontaneität des Verstandes, wobei das erkennende Subjekt Rezeptivität und Spontaneität schon zu synthetischer Einheit gebracht hat.

Was bedeutet dieses Resultat für uns und welche Schlüsse sollten wir daraus ziehen?

16 Ebd., 186c-186e (= S. 137/139). 


\section{Konklusionen aus dem Vergleich von Skandha-Lehre und platonisch inspirierter Bestimmung der Sinnes- und Seelentätigkeit}

Sollten wir den Schluss ziehen, dass eine der beiden porträtierten Auffassungen als falsch anzusehen ist? Oder lassen wir (die in gewisser Weise äußerst unphilosophische) Großzügigkeit walten und belassen es bei zweierlei Wahrheiten? Handelt es sich überhaupt um konkurrierende Zugänge? Oder haben wir etwa eine gar nicht zu überwindende Widersprüchlichkeit offengelegt?

Im Rinzai-Zen gibt es das Üben mit Koans. Aus unseren Überlegungen wäre vielleicht folgender Koan-Spruch ableitbar:

Nimm wahr in absoluter Selbstlosigkeit!

Nimm wahr als absolutes Selbst!

Ich möchte dies so stehen lassen und ich möchte noch so viel Weiteres hierzu erforschen und zur Sprache bringen.

Auf dem zweiten Weg ließe sich sagen: Die Skandha-Lehre ist auch AnatmanLehre. Was heißt jedoch Nicht-Selbst?

Eine von mir sehr geschätzte Interpretation stammt vom vietnamesischen (mit seiner Sangha-Gemeinschaft in Frankreich lebenden) Zenmeister Thich Nhat Hanh. Für ihn offenbart sich im tiefen Verstehen des Anatnam die wechselseitige Durchdringung und Verbindung von allem Seienden ineinander und miteinander. Er nennt es das Intersein, ${ }^{17}$ womit ein nicht bloß ontologischer, sondern zugleich ontopraktischer Ausdruck gefunden ist:

\section{Intersein}

Wenn wir tief in eine Blume hineinschauen, sehen wir all die Elemente, die zusammengekommen sind, um sie hervorzubringen. Wir sehen die Wolken, den Regen. Ohne Regen kann nichts wachsen. Wenn ich die Blume berühre, berühre ich die Wolke und den Regen. Dies ist keine Poesie, dies ist die Wirklichkeit. Wenn wir die Wolke und den Regen aus der Blume herausnehmen wollten, dann wäre die Blume nicht mehr da. Mit den Augen des Buddha sehen wir die Wolken und den Regen in der Blume. Wir können die Sonne berühren, ohne uns die Finger zu verbrennen. Ohne die Sonne kann nichts wachsen, und so können wir die Sonne nicht aus der Blume entfernen. Die Blume kann nicht getrennt existieren, sie muss mit dem Licht, mit den Wolken, mit dem Regen intersein. Das Wort Intersein kommt der Wirklichkeit näher als der Begriff Sein. Sein heißt tatsächlich Intersein.

17 Im Englischen lautet der Terminus „Interbeing“. 
Dasselbe gilt für Sie, für mich. Wir sollten uns darin üben, in unserem Alltag die Wirklichkeit des Interseins, des Nicht-Selbst in jedem Moment zu berühren. Wir sind mit den Wolken, mit dem Regen, mit den Kindern, mit den Bäumen, mit den Flüssen verbunden. Diese Verbindung offenbart uns die wahre Natur der Wirklichkeit, die Natur der Unbeständigkeit, des Interseins, des Nicht-Selbst, der gegenseitigen Abhängigkeit. ${ }^{18}$

Nehmen wir Thich Nhat Hanhs Worte ernst: Dies ist keine Poesie!

Philosophisch müssen wir versuchen, diejenige Position ausfindig zu machen, die in prosaischer Weise am ehesten diese Intersein-Auffassung zum Ausdruck bringt. Meines Erachtens gibt es hier die größte Übereinstimmung mit der Philosophie Hegels. Kein anderer Denker in der abendländischen Tradition hat in so ausführlicher und gehaltvoller Art die These von der Allverbundenheit nicht nur behauptet, sondern systematisch durchgearbeitet und in den großen Sphären der spekulativen Logik, der Naturphilosophie und der Geistphilosophie dargestellt.

Der zentrale Faktor in der Bestimmung, Entfaltung und dialektischen Entwicklung aller philosophischen Themen ist hierbei die Negation bzw. Negativität. Es gibt keine größere Verbindungskraft, worin alle Entitäten in ihrer Bezüglichkeit auf sich und in ihrer Beziehung auf Anderes bestimmt sind, als die Kraft der Negation. Ausschließlich über negative Konstitution (Anderes nicht zu sein) ist jegliche Bestimmung das, was sie ist. Es gibt keine aus dem negativen Gesamtzusammenhang herausgelösten Positivitäten, die ohne prinzipielle Beziehung zu allem Anderen bestehen könnten. Hier besteht eine wunderbare Entsprechung zum sogenannten Intersein.

Wie steht es aber mit dem Nicht-Selbst?

Das Eigenartige bei Hegel ist, dass bei ihm - freilich unwissentlich - alle Konsequenzen aus der Anatman-Lehre (die auch die Grundlage für die SkandhaLehre ist) gezogen sind und philosophisch immanent ausgelegt werden. Andererseits findet sich bei ihm auch eine sehr ausgeklügelte Theorie des Selbst, sogar des absoluten Selbst. Hegel schafft es also, die essentiellen Gehalte der buddhistischen Anatman-Lehre, die er wohl nur peripher kannte, mit der platonischen und nachplatonischen Philosophietradition, die stets an der Konzeption der Seele, der Person, der substantial aufgefassten Individualität usf. orientiert gewesen ist, zu verbinden.

Für mich selbst stellt es eine stetige Auseinandersetzung dar, die buddhistische Lehre und die mit ihr einhergehende Orthopraxis in Beziehung zu den Inhalten des westlichen Philosophiekanons zu setzen, um daraus möglichst tiefgründige und tief begründete Einsichten zu gewinnen. Darum werde ich mich auch weiterhin bemühen. Die obigen Ausführungen sind auch Resultat dieser Anstrengung.

Thich Nhat Hanh 2011, S. 132 f. 


\section{Literaturverzeichnis}

Aitken, Robert: Zen als Lebenspraxis. Mit einem Vorwort von Gary Snyder. Aus dem Amerikanischen von Christian Quatmann. München 1998.

Diogenes Laertius: Leben und Meinungen berühmter Philosophen. In der Übersetzung von Otto Apelt unter Mitarbeit von Hans Günter Zekl neu herausgegeben sowie mit Vorwort, Einleitung und neuen Anmerkungen versehen von Klaus Reich. Zweiter Band. Hamburg ${ }^{3} 1998$.

Eliade, Mircea: Geschichte der religiösen Ideen. Band 2: Von Gautama Buddha bis zu den Anfängen des Christentums. Freiburg im Breisgau ${ }^{2} 1979$.

Izutsu, Toshihiko: Philosophie des Zen-Buddhismus. Aus dem Englischen übersetzt von D. Rosenstein. Reinbek bei Hamburg 1979.

Nyanatiloka: Buddhistisches Wörterbuch. Kurzgefaßtes Handbuch der buddhistischen Lehren und Begriffe in alphabetischer Anordnung. Stammbach-Herrnschrot ${ }^{5} 1999$.

Nyanatiloka: Der Weg zur Erlösung (= Buddhistische Handbibliothek 8). Konstanz ${ }^{2} 1981$.

Platon: „Theaitetos“, in: Platon. Werke in acht Bänden. Griechisch und Deutsch. Herausgegeben von Gunther Eigler. Bearbeitet von Peter Staudacher. Griechischer Text von August Diès. Deutsche Übersetzung von Friedrich Schleiermacher. Sechster Band. Darmstadt ${ }^{2} 1990$.

Thich Nhat Hanh: Im Hier und Jetzt zuhause sein. Freiburg im Breisgau ${ }^{2} 2011$. 